\title{
Preparing diagnostics for long pulse operation at W7-X
}

\author{
Ulrich Neuner ${ }^{\mathrm{a}}$, Bertram Brucker ${ }^{\mathrm{a}}$, Antonio Cardella ${ }^{\mathrm{b}}$, Michael Endler ${ }^{\mathrm{a}}$, Klaus Grosser ${ }^{\mathrm{a}}$, Dag \\ Hathiramani ${ }^{\mathrm{a}}$, Matthias Hirsch ${ }^{\mathrm{a}}$, Ralf König ${ }^{\mathrm{a}}$, Ekkehard Pasch ${ }^{\mathrm{a}}$, Dirk Pilopp ${ }^{\mathrm{a}}$, Matthias Schülke ${ }^{\mathrm{a}}$, \\ Stefan Thiel ${ }^{\mathrm{a}}$, Henning Thomsen ${ }^{\mathrm{a}}$, Robert Wolf ${ }^{\mathrm{a}}$, Daihong Zhang ${ }^{\mathrm{a}}$, the W7-X team \\ ${ }^{\mathrm{a}}$ Max-Planck-Institut für Plasmaphysik EURATOM Association, Greifswald, Germany \\ ${ }^{\mathrm{b}}$ European Commision c/o W7-X, Max-Planck-Institut für Plasmaphysik EURATOM Association, Greifswald, Germany
}

\begin{abstract}
Long pulse operation considerably increases the thermal load on in-vessel components. Diagnostic frontends formerly employed at short pulse machines therefore have to be considerably re-designed for installation in the stellarator W7-X that is currently being built at Greifswald, Germany. The strategy applied to cope with the thermal load is threefold: to reduce the influx of heat on the component, to conduct the heat inside the component to suitable heat sinks and to choose suitable materials for sensitive components. The first is achieved by the shielding against microwave stray radiation, plasma radiation, thermal radiation and particle fluxes and by absorbing residual microwave stray radiation in the immediate vicinity of sensitive components. The second task, suitable heat conduction, enforces severe restrictions on the use of any thin parts like foils or meshes. Thirdly, in order for a component to survive the residual loads, materials must be chosen that absorb only a small fraction of the microwave stray radiation flux, conduct heat well enough, and survive high temperatures and large temperature gradients. Examples are provided from bolometry, magnetic diagnostics, soft X-ray diagnostics and Thomson scattering. Measurements of microwave stray radiation effects are presented, in particular the effectiveness of several shielding concepts.
\end{abstract}

Keywords: Diagnostics, heat load, microwave stray radiation.

\section{Task}

Long pulse operation considerably increases the thermal load on in-vessel components [1]. Diagnostic frontends formerly employed at short pulse machines therefore have to be considerably re-designed [2] for installation in the stellarator W7-X that is currently being built at Greifswald, Germany [3].

Experiments of up to $30 \mathrm{~min}$. duration with up to $10 \mathrm{MW}$ of continuous ECRH power are foreseen.

Heat loads from the following sources need to be considered:

plasma radiation;

particles from the plasma and NBI heating;

thermal radiation from first wall tiles;

microwave stray radiation from ECRH.

\section{MISTRAL - the microwave stray radiation test chamber}

With a length of $2.2 \mathrm{~m}$, a diameter of $1.48 \mathrm{~m}$ and several ports, the largest of which measures $0.4 \mathrm{~m} \times 1 \mathrm{~m}$, the chamber, shown in figure 1 , can test all equipment that is to be installed inside $\mathrm{W} 7-\mathrm{X}$.

Components will typically be tested at microwave stray radiation levels of $50 \mathrm{~kW} / \mathrm{m}^{2}$. The frequency is $140 \mathrm{GHz}$ and the duration is up to 30 minutes.

Diagnostics for the tests comprise graphite bolometers to measure the microwave level, pressure sensors, a mass spectrometer, a video camera, an infrared camera and thermo couples [4].

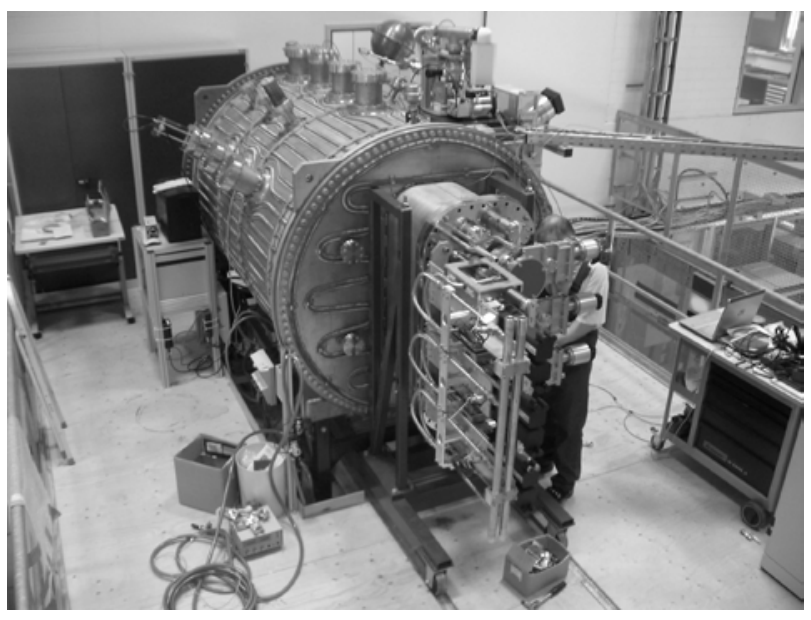

Fig. 1. The microwave stray radiation test chamber.

Measurements in the chamber were performed to test materials, concepts and components, examples of which are described in sections 3 to 5 .

\section{Reduction of heat influx}

\subsection{Partial shielding}

For some sturdy diagnostic frontends, a slight reduction of the plasma radiation and the microwave 
stray radiation suffices. The quartz window of the Thomson scattering observation optics, for example, has been tested to withstand a total power influx of $10 \mathrm{~kW} / \mathrm{m}^{2}$. This is below the guideline value for components in the vessel, which is $50 \mathrm{~kW} / \mathrm{m}^{2}$. However, since it is somewhat retracted in its port, a reduction of the size of the opening in the first wall by a factor of 2 is all that is needed for the power level at the actual position of the window to be acceptable.

Other optical diagnostics compensate for being positioned closer to the plasma than the front window of the Thomson scattering observation optics by having smaller entrance apertures.

Heat shields to block off direct thermal radiation from the back side of hot first wall tiles are envisaged for some magnetic diagnostics positioned between the first wall and the plasma vessel.

\subsection{Complete shielding}

Cables and magnetic diagnostics like Rogowski coils can be completely covered in microwave tight metal casings (with $0.7 \mathrm{~mm}$ holes for vacuum pumping). However, the electrical conductivity of diagnostic casings must remain low (to keep the time resolution within acceptable limits) while thermal conduction must remain high (for a solution see section 4), thus imposing severe restrictions on the choice of materials and the thickness of the casing.

For the Rogowski coils, steel tubes of $0.25 \mathrm{~mm}$ wall thickness were chosen. The diamagnetic loops are designed with a casing of thin copper, partly with a galvanic separation on the circumference.

For the signal cables the conductivity restriction does not apply. All signal cables with organic insulation will therefore be routed inside copper tubes (with $0.7 \mathrm{~mm}$ diameter holes for vacuum pumping).

\subsection{Absorption}

Microwave stray radiation can be absorbed to reduce its influence on diagnostics. This is done at the bolometer, which is designed as a pin hole camera. Entrance aperture, absorber $\left(150 \mu \mathrm{m}_{2} \mathrm{O}_{3} / \mathrm{TiO}_{2}\right.$ coating of the inner surfaces) and screening (wire-mesh or perforated foil in front of the detector) result in a microwave damping by a factor of 300 as compared to a set-up with the aperture alone.

This large reduction factor is needed, since the bolometer cannot distinguish between the desired signal caused by heating of the gold foil sensor by plasma radiation and the unwanted signal caused by heating of the sensor by microwave stray radiation.

\subsection{Reflection}

Measurements with a bellows (later to be employed in the soft X-ray camera system) in the microwave stray radiation test chamber showed that coating the steel bellows with reflecting copper reduces the heating by microwaves, but also reduces the cooling by thermal radiation. For 30 minute discharges, the two effects nearly cancel out and almost no net reduction in the maximum temperature could be achieved.

\section{Heat conduction}

Over the course of long pulse discharges, even highly reflective objects heat up and need thermal coupling to water cooled components.

An example is the diamagnetic loop, which is completely covered in a copper casing. Exposed to the microwave stray radiation its temperature increased quickly enough that the upper temperature limit was reached after 18 minutes. Lengthening this period to the required 30 minutes was only possible by increasing the thermal conduction to the water cooled vessel wall. To achieve this, copper strips were added to the fastening steel bolts.

The Rogowski coils need heat conduction along the coils to the fixings, where heat can be conducted to the vessel wall. To increase the heat conduction of the coils and the thin walled steel casing, T-shaped copper profiles were introduced between the casings.

Thin components like wires, meshes or foils are particularly unsuited to conduct heat and consequentially their temperature rises quickly even for modest incident heat fluxes. Consequently, they are employed only in very sheltered areas like the inside of the bolometer pinhole cameras or inside the shielding tubes for cables.

\section{Choice of materials}

Where shielding is not possible, some components simply have to take large heat loads.

This is true for the Mirnov coils. Candidates for the winding core were Boron Nitride and Aluminum Nitride (BN with $20 \% \mathrm{ZrO}$ proved to be too brittle). While porous $\mathrm{BN}$ is much easier to machine and therefore much lower in total costs, AlN has a much better heat conduction (heat conductivity of AlN: $180 \mathrm{~W} /(\mathrm{m} \mathrm{K})$, heat conductivity of porous $\mathrm{BN}$ : $23 \mathrm{~W} /(\mathrm{m} \mathrm{K})$ ) and is therefore favored.

The use of a mineral insulated wire relieves the coils from the necessity of individual grooves to fix the winding, as shown in figure 2. With this design simplification, the manufacturing effort could be kept within acceptable limits. 


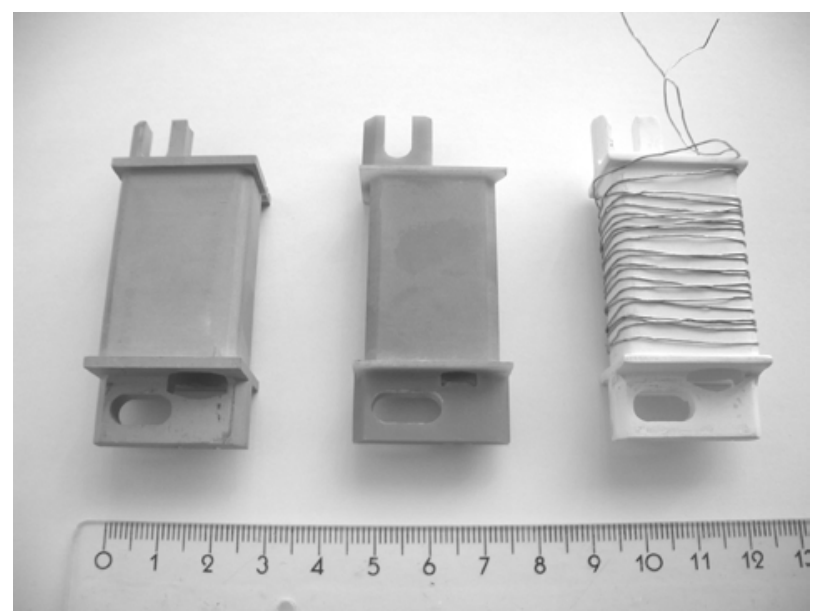

Fig. 2. BN with $20 \% \mathrm{ZrO}$, AlN and BN winding cores for the Mirnov coils.

\section{Conclusions}

Long pulse operation under fusion relevant conditions constitutes a harsh environment for delicate diagnostics. Several heat sources raise the temperature of every component in the vessel. A combination of reducing the influx of heat on the components, conducting the heat inside the components to suitable heat sinks and choosing suitable materials for sensitive components is mandatory. In doing so, each diagnostic requires its own specific solution.

\section{Acknowledgments}

We wish to express our thanks to all persons from the W7-X team involved in the measurements and design work presented in this article. In this paper are expressed only personal opinions. These do not oblige the European Commission in any way.

\section{References}

[1] H.-J. Hartfuss et al., Diagnostics for steady state plasmas, Plasma Phys. Control. Fusion 48, R83 (2006).

[2] R. Burhenn et al., Diagnostic Development for Steady State Operation of the Stellarator Wendelstein 7-X, Contrib. Plasma Phys. 51 (2-3) (2011) 271-278.

[3] St. Bosch et al., Construction of Wendelstein 7-XEngineering a Steady-State Stellarator, IEEE Transactions on Plasma Science 38 (3) (2010) 265-273.

[4] M. Hirsch et al., Qualifying Plasma Diagnostics for a high-power microwave background of ECR-heated discharges, Proceedings of the International Conference on Plasma Diagnostics April 12-16, 2010, Pont-à-Mousson, France. 